\title{
Numerical approximation of the space-time Caputo-Fabrizio fractional derivative and application to groundwater pollution equation
}

\author{
Abdon Atangana' and Rubayyi T Alqahtani ${ }^{2 *}$
}

\author{
"Correspondence: \\ rtalqahtani@imamu.edu.sa \\ ${ }^{2}$ Department of Mathematics and \\ Statistics, College of Science, \\ Al-Imam Mohammad Ibn Saud \\ Islamic University (IMSIU), Riyadh, \\ 11566, Saudi Arabia \\ Full list of author information is \\ available at the end of the article
}

\begin{abstract}
Recently, Caputo and Fabrizio proposed a new derivative with fractional order without singular kernel. The derivative has several interesting properties that are useful for modeling in many branches of sciences. For instance, the derivative is able to describe substance heterogeneities and configurations with different scales. In order to accommodate researchers dealing with numerical analysis, we propose a numerical approximation in time and space. We modified the advection dispersion equation by replacing the time derivative with the new fractional derivative. We solve numerically the modified equation using the proposed numerical approximation. The stability and convergence analysis of the numerical scheme were presented together with some simulations.
\end{abstract}

Keywords: Caputo-Fabrizio derivative; numerical approximation; advection diffusion equation; stability analysis

\section{Introduction}

In the last decade, many physical problems have been modeled using the concept of noninteger-order derivative. These derivatives of fractional order range from RiemannLiouville via Caputo to Caputo-Fabrizio [1, 2]. We can find in the literature many analytical approaches to deal with differential equations with fractional equations [3-10]. Most of these techniques are dealing with linear fractional differential equations. However, most fractional differential equations describing real-world problems are highly complicated and cannot sometime be handled via analytical methods. In order to solve these problems in many cases, researchers rely on the use of numerical methods because these problems have initial conditions, boundary condition, and source terms that turn hard to find an analytical solution.

Several numerical approaches in connection with derivatives of fractional order describing real-world problems alter essentially in the many in which the derivative of fractional order is tailored [11-24]. Approximation representation of a derivative of fractional order has a highly complicated formula compared to those of integer order because fractional derivatives are nonlocal, and therefore the calculation at a particular point requires knowl-

(c) 2016 Atangana and Alqahtani. This article is distributed under the terms of the Creative Commons Attribution 4.0 International License (http://creativecommons.org/licenses/by/4.0/), which permits unrestricted use, distribution, and reproduction in any medium, provided you give appropriate credit to the original author(s) and the source, provide a link to the Creative Commons license, and indicate if changes were made. 
edge of the function further out of the region close to that point. Accordingly, finite difference approximations of derivatives of fractional order engage a quantity of points that alters according to how faraway we are from the border line [12-14].

The most recent derivative of fractional order was proposed by Caputo and Fabrizio [1], who demonstrated that the new-fangled derivative encompasses extra encouraging properties in comparison with the old version. They demonstrated, for example, that it can depict substance heterogeneities and configurations with different scales, which obviously cannot be overseeing with the prominent local theories and also the well-known fractional derivative. An additional application is in the investigation of the macroscopic behaviors of some materials, associated with nonlocal communications between atoms, which are recognized to be important of the properties of material. We present the definition of the Caputo fractional derivative.

Definition 1 The Caputo derivative of fractional order old editor of a function $f$ is given as

$$
{ }_{0}^{C} D_{x}^{\alpha}(f(x))=\frac{1}{\Gamma(n-\alpha)} \int_{0}^{x}(x-t)^{n-\alpha-1} \frac{d^{n}}{d t^{n}}(f(t)) d t, \quad n-1<\alpha \leq n .
$$

Definition 2 Let $f \in H^{1}(a, b), b>a, \alpha \in[0,1]$. Then the new Caputo fractional derivative is defined as

$$
{ }_{0}^{C F} D_{t}^{\alpha}(f(t))=\frac{M(\alpha)}{1-\alpha} \int_{a}^{t} f^{\prime}(x) \exp \left[-\alpha \frac{t-x}{1-\alpha}\right] d x,
$$

where $M(\alpha)$ is a normalization function such that $M(0)=M(1)=1$ [1]. However, if the function does not belong to $H^{1}(a, b)$ then, the derivative can be redefined as

$$
{ }_{0}^{C F} D_{t}^{\alpha}(f(t))=\frac{\alpha M(\alpha)}{1-\alpha} \int_{a}^{t}(f(t)-f(x)) \exp \left[-\alpha \frac{t-x}{1-\alpha}\right] d x
$$

The aim of this paper is to propose a numerical approximation of the space and time Caputo-Fabrizio derivative of fractional order that will be used by researchers in the field of numerical analysis.

\section{Caputo-Fabrizio approximations}

In this section, we derive a numerical approximation based upon the definition of newly proposed derivative of fractional order [4],

$$
{ }_{0}^{C F} D_{t}^{\alpha}(f(t))=\frac{M(\alpha)}{1-\alpha} \int_{0}^{t} f^{\prime}(x) \exp \left[-\alpha \frac{t-x}{1-\alpha}\right] d x .
$$

For some positive integer $N$, the grid size in time for finite difference technique is defined by

$$
k=\frac{1}{N}
$$

The grid points in the time interval $[0, T]$ are labeled $t_{n}=n k, n=0,1,2, \ldots, T N$. The value of the function $f$ at the grid point is $f_{i}=f\left(t_{i}\right)$. 
A discrete approximation to the Caputo-Fabrizio derivative of fractional order can be obtained by simple quadrature formula as follows:

$$
{ }_{0}^{C F} D_{t}^{\alpha}\left(f\left(t_{n}\right)\right)=\frac{M(\alpha)}{1-\alpha} \int_{0}^{t_{n}} f^{\prime}(x) \exp \left[-\alpha \frac{t_{n}-x}{1-\alpha}\right] d x
$$

This equation can be modified using the first-order approximation to

$$
{ }_{0}^{C F} D_{t}^{\alpha}\left(f\left(t_{j}\right)\right)=\frac{M(\alpha)}{1-\alpha} \sum_{j=1}^{n} \int_{(j-1) k}^{j k}\left(\frac{f^{k+1}-f^{k}}{\Delta t}+O(\Delta t)\right) \exp \left[-\alpha \frac{t_{j}-x}{1-\alpha}\right] d x
$$

Before integration we obtain the following expression

$$
\begin{aligned}
& \frac{M(\alpha)}{1-\alpha} \sum_{j=1}^{n}\left(\frac{f^{j+1}-f^{j}}{\Delta t}+O(\Delta t)\right) \int_{(j-1) k}^{j k} \exp \left[-\alpha \frac{t_{n}-x}{1-\alpha}\right] d x, \\
& { }_{0}^{C F} D_{t}^{\alpha}\left(f\left(t_{j}\right)\right)=\frac{M(\alpha)}{\alpha} \sum_{j=1}^{n}\left(\frac{f^{j+1}-f^{j}}{\Delta t}+O(\Delta t)\right) d_{j, k},
\end{aligned}
$$

where

$$
d_{j, k}=\exp \left[-\alpha \frac{k}{1-\alpha}(n-j+1)\right]-\exp \left[-\alpha \frac{k}{1-\alpha}(n-j)\right]
$$

We finally have that

$$
{ }_{0}^{C F} D_{t}^{\alpha}\left(f\left(t_{n}\right)\right)=\frac{M(\alpha)}{\alpha} \sum_{j=1}^{n}\left(\frac{f^{j+1}-f^{j}}{\Delta t}\right) d_{j, k}+\frac{M(\alpha)}{\alpha} \sum_{j=1}^{n} d_{j, k} O(\Delta t) .
$$

Theorem 1 Let $f(x)$ be a function in $C^{2}[a, b]$, and let the order of the fractional derivative be $0<\alpha \leq 1$. Then the first-order approximation of the Caputo-Fabrizio derivative at a point $t_{n}$ is

$$
{ }_{0}^{C F} D_{t}^{\alpha}\left(f\left(t_{n}\right)\right)=\frac{M(\alpha)}{\alpha} \sum_{j=1}^{n}\left(\frac{f^{j+1}-f^{j}}{\Delta t}\right) d_{j, k}+O\left((\Delta t)^{2}\right)
$$

Proof From equation (8) we have

$$
\begin{aligned}
{ }_{0}^{C F} D_{t}^{\alpha}\left(f\left(t_{n}\right)\right)= & \frac{M(\alpha)}{\alpha} \sum_{j=1}^{n}\left(\frac{f^{j+1}-f^{j}}{\Delta t}\right) d_{j, k} \\
& +\frac{M(\alpha)}{\alpha} \sum_{j=1}^{n}\left(\exp \left[-\alpha \frac{k}{1-\alpha}(n-j+1)\right]-\exp \left[-\alpha \frac{k}{1-\alpha}(n-j)\right]\right) O(\Delta t) .
\end{aligned}
$$

However,

$$
\sum_{j=1}^{n}\left(\exp \left[-\alpha \frac{k}{1-\alpha}(n-j+1)\right]-\exp \left[-\alpha \frac{k}{1-\alpha}(n-j)\right]\right)=\exp \left[-\alpha \frac{k}{1-\alpha}(n)\right]-1
$$


Now the approximation of the exponential function can be obtained as

$$
\exp \left[-\alpha \frac{k}{1-\alpha}(n)\right] \approx 1-\alpha \frac{k}{1-\alpha}(n)
$$

Then replacing the above in equation (11), we obtain

$$
\sum_{j=1}^{n}\left(\exp \left[-\alpha \frac{k}{1-\alpha}(n-j+1)\right]-\exp \left[-\alpha \frac{k}{1-\alpha}(n-j)\right]\right) \approx-\alpha \frac{k}{1-\alpha}(n) .
$$

Then equation (11) becomes

$$
{ }_{0}^{C F} D_{t}^{\alpha}\left(f\left(t_{n}\right)\right)=\frac{M(\alpha)}{\alpha} \sum_{j=1}^{n}\left(\frac{f^{j+1}-f^{j}}{\Delta t}\right) d_{j, k}+\frac{M(\alpha) k}{1-\alpha}(n) O(\Delta t) .
$$

We therefore obtain the requested result

$$
{ }_{0}^{C F} D_{t}^{\alpha}\left(f\left(t_{n}\right)\right)=\frac{M(\alpha)}{\alpha} \sum_{j=1}^{n}\left(\frac{f^{j+1}-f^{j}}{\Delta t}\right) d_{j, k}+O\left((\Delta t)^{2}\right) .
$$

This completes the proof.

We now conclude that the first-order approximation method for the computation of the Caputo-Fabrizio derivative of time fractional order is given as

$$
{ }_{0}^{C F} D_{t}^{\alpha}\left(f\left(t_{n}\right)\right)=\frac{M(\alpha)}{\alpha} \sum_{j=1}^{n}\left(\frac{f^{j+1}-f^{j}}{\Delta t}\right) d_{j, k} .
$$

We next propose the first order for the space fractional order.

For some positive integer $N$, the grid sizes in time for finite difference technique is defined by

$$
i=\frac{1}{M}
$$

The grid points in the time interval $[0, X]$ are labeled $x_{i}=m i, m=0,1,2, \ldots, X M$.

The value of the function $f$ at the grid point is $f_{i}^{k}=f\left(x_{i}, t_{k}\right)$. We have

$$
{ }_{0}^{C F} D_{t}^{\alpha}\left(f\left(x_{m}, t_{i}\right)\right)=\frac{M(\alpha)}{\sqrt{\pi}(1-\alpha)} \int_{0}^{x_{m}} \frac{\partial}{\partial y} f^{\prime}\left(y, t_{i}\right) \exp \left[-\alpha^{2} \frac{\left(x_{m}-y\right)^{2}}{(1-\alpha)^{2}}\right] d y .
$$

Now employing the Crank-Nicolson approximation for the first-order derivative, the above equation is converted to

$$
\begin{aligned}
{ }_{0}^{C F} D_{x}^{\alpha}\left(f\left(x_{m}, t_{k}\right)\right)= & \frac{M(\alpha)}{\sqrt{\pi}(1-\alpha)} \int_{0}^{x_{m}}\left(\frac{\left(f_{i+1}^{k+1}-f_{i-1}^{k+1}\right)-\left(f_{i+1}^{k}-f_{i-1}^{k}\right)}{4 \Delta x}+O(\Delta t)\right) \\
& \times \exp \left[-\alpha^{2} \frac{\left(x_{m}-y\right)^{2}}{(1-\alpha)^{2}}\right] d y .
\end{aligned}
$$


The latter equation can be converted to

$$
\begin{aligned}
{ }_{0}^{C F} D_{x}^{\alpha}\left(f\left(x_{m}, t_{i}\right)\right)= & \frac{M(\alpha)}{\sqrt{\pi}(1-\alpha)} \sum_{l=1}^{m}\left\{\frac{\left(f_{l+1}^{k+1}-f_{l-1}^{k+1}\right)-\left(f_{l+1}^{k}-f_{l-1}^{k}\right)}{4 \Delta x}+O(i)\right\} \\
& \times \int_{(l-1) i}^{l i} \exp \left[-\alpha^{2} \frac{(i m-y)^{2}}{(1-\alpha)^{2}}\right] d y,
\end{aligned}
$$

where the integral part is given as

$$
\begin{aligned}
& \int_{(l-1) i}^{l i} \exp \left[-\alpha^{2} \frac{(i m-y)^{2}}{(1-\alpha)^{2}}\right] d y \\
& \quad=\frac{(1-\alpha) \sqrt{\pi}}{2 \alpha}\left\{\operatorname{erf}\left[(m i-l i) \frac{\alpha}{1-\alpha}\right]-\operatorname{erf}\left[(m i-l i+i) \frac{\alpha}{1-\alpha}\right]\right\},
\end{aligned}
$$

so that equation (15) becomes

$$
\begin{aligned}
{ }_{0}^{C F} D_{x}^{\alpha}\left(f\left(x_{m}, t_{k}\right)\right) \\
=\frac{M(\alpha)}{1-\alpha} \sum_{l=1}^{m}\left\{\frac{\left(f_{l+1}^{k+1}-f_{l-1}^{k+1}\right)-\left(f_{l+1}^{k}-f_{l-1}^{k}\right)}{4 \Delta x}+O(i)\right\} \\
\quad \times \frac{(1-\alpha)}{2 \alpha}\left\{\operatorname{erf}\left[(m-l) \frac{\alpha i}{1-\alpha}\right]-\operatorname{erf}\left[(m-l+1) \frac{\alpha i}{1-\alpha}\right]\right\} .
\end{aligned}
$$

From the above we obtain

$$
\begin{aligned}
{ }_{0}^{C F} D_{x}^{\alpha}\left(f\left(x_{m}, t_{k}\right)\right) \\
=\frac{M(\alpha)}{1-\alpha} \sum_{l=1}^{m}\left\{\frac{\left(f_{l+1}^{k+1}-f_{l-1}^{k+1}\right)-\left(f_{l+1}^{k}-f_{l-1}^{k}\right)}{4 \Delta x} \frac{(1-\alpha) \sqrt{\pi}}{2 \alpha}\right. \\
\left.\quad \times\left\{\operatorname{erf}\left[(m-l) \frac{\alpha i}{1-\alpha}\right]-\operatorname{erf}\left[(m-l+1) \frac{\alpha i}{1-\alpha}\right]\right\}\right\} \\
+O(i) \frac{(1-\alpha)}{2 \alpha} \sum_{l=1}^{m}\left\{\operatorname{erf}\left[(m-l) \frac{\alpha i}{1-\alpha}\right]-\operatorname{erf}\left[(m-l+1) \frac{\alpha i}{1-\alpha}\right]\right\}
\end{aligned}
$$

Theorem 2 Let $f(x, t)$ be a function in $C^{2}([a, b] \times[0, T])$, and let the order of the fractional derivative be $0<\alpha \leq 1$. Then the first-order approximation of the Caputo-Fabrizio derivative at a point $\left(x_{m}, t_{n}\right)$ is

$$
{ }_{0}^{C F} D_{x}^{\alpha}\left(f\left(x_{m}, t_{k}\right)\right)=\frac{M(\alpha)}{2 \alpha} \sum_{l=1}^{m}\left\{\frac{\left(f_{l+1}^{k+1}-f_{l-1}^{k+1}\right)-\left(f_{l+1}^{k}-f_{l-1}^{k}\right)}{4 \Delta x}\right\} d_{i, l}+R(\alpha, i, l)
$$

where

$$
d_{i, l}=\left\{\operatorname{erf}\left[(m-l) \frac{\alpha i}{1-\alpha}\right]-\operatorname{erf}\left[(m-l+1) \frac{\alpha i}{1-\alpha}\right]\right\}, \quad\|R(\alpha, i, l)\|<M
$$


Proof From equation (23) we have that

$$
\begin{aligned}
{ }_{0}^{C F} D_{x}^{\alpha}\left(f\left(x_{m}, t_{k}\right)\right) \\
=\frac{M(\alpha)}{2 \alpha} \sum_{l=1}^{m}\left\{\frac { ( f _ { l + 1 } ^ { k + 1 } - f _ { l - 1 } ^ { k + 1 } ) - ( f _ { l + 1 } ^ { k } - f _ { l - 1 } ^ { k } ) } { 4 \Delta x } \left\{\operatorname{erf}\left[(m-l) \frac{\alpha i}{1-\alpha}\right]\right.\right. \\
\left.\left.\quad-\operatorname{erf}\left[(m-l+1) \frac{\alpha i}{1-\alpha}\right]\right\}\right\} \\
\quad+O(i) \frac{M(\alpha)}{2 \alpha} \sum_{l=1}^{m}\left\{\operatorname{erf}\left[(m-l) \frac{\alpha i}{1-\alpha}\right]-\operatorname{erf}\left[(m-l+1) \frac{\alpha i}{1-\alpha}\right]\right\} .
\end{aligned}
$$

We put

$$
R(\alpha, i, l)=O(i) \frac{M(\alpha)}{2 \alpha} \sum_{l=1}^{m}\left\{\operatorname{erf}\left[(m-l) \frac{\alpha i}{1-\alpha}\right]-\operatorname{erf}\left[(m-l+1) \frac{\alpha i}{1-\alpha}\right]\right\}
$$

Then taking the norm to both sides, we have

$$
\begin{aligned}
& \|R(\alpha, i, l)\|=\| O(i) \frac{M(\alpha)}{2 \alpha} \sum_{l=1}^{m}\left\{\operatorname{erf}\left[(m-l) \frac{-\alpha i}{1-\alpha}\right]-\operatorname{erf}\left[(m-l+1) \frac{-\alpha i}{1-\alpha}\right]\right\}, \\
& \|R(\alpha, i, l)\|=\left\|O(i) \frac{M(\alpha)}{2 \alpha}\left(\operatorname{erf}\left[m \frac{-\alpha i}{1-\alpha}\right]\right)\right\| .
\end{aligned}
$$

This completes the proof.

Then, the first-order approximation method for the computation of Caputo-Fabrizio derivative of space fractional order is given as

$$
\begin{aligned}
{ }_{0}^{C F} D_{x}^{\alpha}\left(f\left(x_{m}, t_{i}\right)\right)= & \frac{M(\alpha)}{2 \alpha} \sum_{l=1}^{m}\left\{\frac{\left(f_{l+1}^{k+1}-f_{l-1}^{k+1}\right)-\left(f_{l+1}^{k}-f_{l-1}^{k}\right)}{4 \Delta x}\right. \\
& \left.\times\left\{\operatorname{erf}\left[(m-l) \frac{\alpha i}{1-\alpha}\right]-\operatorname{erf}\left[(m-l+1) \frac{\alpha i}{1-\alpha}\right]\right\}\right\} .
\end{aligned}
$$

\section{Application to some well-known equations}

In this section, we present a numerical solution of the time fractional advection diffusion equation in heterogeneous medium. The fractional derivative used here is of the CaputoFabrizio type.

The reason of this modification is that the fractional derivatives are recollection operational which recurrently distinguish indulgence of force or damage in the passable as in the case of inelastic media or reconsideration of the porosity in the thinning out in permeable media and supplementary in comprehensive they are in traditional values throughout the subsequent theory of hydrology. They are accredited not merely for the motivation that they match appropriately a variety of noticeable actuality, nevertheless, additionally for the motive that they own the well-designed alongside with scrupulous property that although the order of differentiation is integer, they match by means of the traditional derivative of that order. On the other hand, this chattel is not pertinent to the effect they characterize in 
the physical observable fact and conjectures if using other differential operators, probably simpler nevertheless devoid of this property, one may get similar responds of fractional order derivative. Therefore, in order to well replicate the flow of the particles via porous media in different scale in the medium, we replace the ordinary derivative in time with the scale time derivative proposed by Caputo and Fabrizio. The equation under consideration here is

$$
{ }_{0}^{C F} D_{t}^{\alpha}(P(x, t))+\frac{\varphi u}{c_{\rho}} \frac{\partial P(x, t)}{\partial x}-\frac{\lambda}{c_{\rho}} \frac{\partial^{2} P(x, t)}{\partial x^{2}}=\frac{Q(x, t)}{c_{\rho}} .
$$

In equation (27), four terms represents transient, advection, and source terms, respectively, $P(x, t)$ is the particle, heat, pollution, or other physical quantities; $c$ is the specific of heat, particles, or other physical quantities; $\varphi$ is the porosity that is the ratio of the liquid volume to the total volume of the medium via which the flow is taken place, $\rho, \lambda$ are the mass density and thermal conductivity, respectively, and, finally, $Q(x, t)$ is the source term. Now substituting equation (26) into (27), we obtain

$$
\begin{gathered}
\frac{M(\alpha)}{2 \alpha} \sum_{k=1}^{j+1} \frac{P_{i}^{k}-P_{i}^{k-1}}{\tau}\left(\operatorname{erf}\left((j-k) \frac{\alpha k}{1-\alpha}\right)-\operatorname{erf}\left((j-k+1) \frac{\alpha k}{1-\alpha}\right)\right) \\
=\frac{\lambda}{2 h^{2} c_{\rho}}\left\{\left(P_{i+1}^{j+1}-2 P_{i}^{j+1}+P_{i-1}^{j+1}\right)+\left(P_{i+1}^{j}-2 P_{i}^{j}+P_{i-1}^{j}\right)\right\} \\
\quad-\frac{\varphi u}{c_{\rho}}\left\{\left(P_{i+1}^{j+1}-P_{i-1}^{j+1}\right)+\left(P_{i+1}^{j}-P_{i-1}^{j}\right)\right\}+\frac{Q_{i}^{j+1}+Q_{i}^{j}}{2 c_{\rho}} .
\end{gathered}
$$

The above equation can be converted to

$$
\begin{aligned}
\frac{M(\alpha)}{2 \alpha} & \left(\frac{P_{i}^{j+1}-P_{i}^{j}}{\tau}+\sum_{k=1}^{j} \frac{P_{i}^{j+1-k}-P_{i}^{j-k}}{\tau}\right) d_{k, j}^{\alpha} \\
= & \frac{\lambda}{2 h^{2} c_{\rho}}\left\{\left(P_{i+1}^{j+1}-2 P_{i}^{j+1}+P_{i-1}^{j+1}\right)+\left(P_{i+1}^{j}-2 P_{i}^{j}+P_{i-1}^{j}\right)\right\} \\
& \quad-\frac{\varphi u}{4 h c_{\rho}}\left\{\left(P_{i+1}^{j+1}-P_{i-1}^{j+1}\right)+\left(P_{i+1}^{j}-P_{i-1}^{j}\right)\right\}+\frac{Q_{i}^{j+1}+Q_{i}^{j}}{2 c_{\rho}} .
\end{aligned}
$$

For simplicity, let us put

$$
a=\frac{M(\alpha)}{2 \alpha \tau}, \quad b=\frac{\lambda}{2 h^{2} c_{\rho}}, \quad c=\frac{\varphi u}{4 h c_{\rho}} .
$$

Rearranging, we obtain the following recursive formula:

$$
\begin{aligned}
\left(a d_{k, j}^{\alpha}+2 b\right) P_{i}^{j+1}= & \left(a d_{k, j}^{\alpha}-2 b\right) P_{i}^{j}+a \sum_{k=1}^{j}\left(P_{i}^{j+1-k}-P_{i}^{j-k}\right) d_{k, j}^{\alpha} \\
& +b\left\{\left(P_{i+1}^{j+1}+P_{i-1}^{j+1}\right)+\left(P_{i+1}^{j}+P_{i-1}^{j}\right)\right\}-c\left\{\left(P_{i+1}^{j+1}-P_{i-1}^{j+1}\right)+\left(P_{i+1}^{j}-P_{i-1}^{j}\right)\right\} \\
& +\frac{Q_{i}^{j+1}+Q_{i}^{j}}{2 c_{\rho}}
\end{aligned}
$$




\section{Stability analysis of the numerical scheme}

We present in this section the stability analysis of the Crank-Nicolson scheme for time fractional advection diffusion equation. For this, we let $e_{i}^{j}=P_{i}^{j}-p_{i}^{j}$ with $p_{i}^{j}$ the approximate solution at the point $\left(x_{i}, t_{j}\right)(i=1,2, \ldots, N, j=1,2,3, \ldots, M)$ and, as usual, $e^{j}=\left[e_{1}^{j}, \ldots, e_{N}^{j}\right]^{T}$. The error committed while solving the time fractional advection diffusion equation with the Crank-Nicolson scheme is

$$
\begin{aligned}
\left(a d_{k, j}^{\alpha}+2 b\right) e_{i}^{j+1}= & \left(a d_{k, j}^{\alpha}-2 b\right) e_{i}^{j}+a \sum_{k=1}^{j}\left(e_{i}^{j+1-k}-e_{i}^{j-k}\right) d_{k, j}^{\alpha} \\
& +b\left\{\left(e_{i+1}^{j+1}+e_{i-1}^{j+1}\right)+\left(e_{i+1}^{j}+e_{i-1}^{j}\right)\right\}-c\left\{\left(e_{i+1}^{j+1}-e_{i-1}^{j+1}\right)+\left(e_{i+1}^{j}-e_{i-1}^{j}\right)\right\} \\
& +\frac{Q_{i}^{j+1}+Q_{i}^{j}}{2 c_{\rho}} .
\end{aligned}
$$

Here, we assume that

$$
e_{i}^{j}=f(j) \exp (\tau \sigma i j)
$$

where $\sigma$ is the real spatial wave number [4]. However, substituting equation (32) into equation (31), we obtain, for $j=0$,

$$
\left(a d_{k, 0}^{\alpha}+4 b \sin ^{2}\left(\frac{\sigma i}{2}\right)\right) f(1)=\left(a d_{k, 0}^{\alpha}-4 b \sin ^{2}\left(\frac{\sigma i}{2}\right)\right) f(0)
$$

and for $j>0$, we have

$$
\begin{aligned}
\left(a d_{k, j}^{\alpha}+4 b \sin ^{2}\left(\frac{\sigma i}{2}\right)\right) f(j)= & \left(a d_{k, j}^{\alpha}-4 b \sin ^{2}\left(\frac{\sigma i}{2}\right)\right) f(j-1) \\
& -a \sum_{l=1}^{j-1} f(j-l) d_{k, j}^{\alpha}+f(j+1) d_{k, 0}^{\alpha} .
\end{aligned}
$$

Theorem 3 Assume that $f(k)$ satisfies equations (33) and (34). Then, for all $k>0$,

$$
|f(j)| \leq|f(0)|
$$

Proof We shall prove this theorem by employing the recursive method on the natural number $j$. Then, when $j=0$, we have equation (34), and we reformulate it as follows:

$$
\left|\frac{f(1)}{f(0)}\right|=\left|\frac{\left(a d_{k, 0}^{\alpha}-4 b \sin ^{2}\left(\frac{\sigma i}{2}\right)\right)}{\left(a d_{k, 0}^{\alpha}+4 b \sin ^{2}\left(\frac{\sigma i}{2}\right)\right)}\right| \leq 1 .
$$

This implies

$$
|f(1)| \leq|f(0)|
$$


The property is verified for $j=0$. Let us assume that this property is also satisfied for any $j \geq 1$. We shall verify that the property holds also for $j+1$ :

$$
\left(a d_{k, j}^{\alpha}+4 b \sin ^{2}\left(\frac{\sigma i}{2}\right)\right) f(j+1)=\left(a d_{k, j}^{\alpha}-4 b \sin ^{2}\left(\frac{\sigma i}{2}\right)\right) f(j)-a \sum_{l=1}^{j} f(j-l) d_{k, l}^{\alpha} .
$$

Now taking the norms of both sides of equation (37), we obtain

$$
\left|a d_{k, j}^{\alpha}+4 b \sin ^{2}\left(\frac{\sigma i}{2}\right)\right| f(j+1)|\leq|\left(a d_{k, j}^{\alpha}-4 b \sin ^{2}\left(\frac{\sigma i}{2}\right)\right)|| f(j)\left|+\sum_{l=1}^{j}\right| f(j-l) \mid d_{k, l}^{\alpha}
$$

Nonetheless, we recall that the property holds up to $j$. Thus, we transform the above equation into

$$
\left|a d_{k, j}^{\alpha}+4 b \sin ^{2}\left(\frac{\sigma i}{2}\right)\right| f(j+1)|\leq|\left(a d_{k, j}^{\alpha}-4 b \sin ^{2}\left(\frac{\sigma i}{2}\right)\right)|| f(0)\left|+\sum_{l=1}^{j}\right| f(0) \mid d_{k, l}^{\alpha} .
$$

Rearranging, we obtain

$$
\begin{aligned}
& \left|a d_{k, j}^{\alpha}+4 b \sin ^{2}\left(\frac{\sigma i}{2}\right)\right||f(j+1)| \\
& \quad \leq\left\{\left|\left(a d_{k, j}^{\alpha}-4 b \sin ^{2}\left(\frac{\sigma i}{2}\right)\right)\right|+\left\{\operatorname{erf}\left[\frac{m \alpha i}{1-\alpha}\right]-\operatorname{erf}\left[\frac{\alpha i}{1-\alpha}\right]\right\}\right\}|f(0)| .
\end{aligned}
$$

It is important to recall that

$$
|\operatorname{erf}[x]| \leq 1, \quad \operatorname{erf}\left[\frac{m \alpha i}{1-\alpha}\right]-\operatorname{erf}\left[\frac{\alpha i}{1-\alpha}\right] \leq 0 .
$$

Therefore,

$$
\frac{|f(j+1)|}{|f(0)|} \leq\left|\frac{\left|\left(a d_{k, j}^{\alpha}-4 b \sin ^{2}\left(\frac{\sigma i}{2}\right)\right)\right|+\left\{\operatorname{erf}\left[\frac{m \alpha i}{1-\alpha}\right]-\operatorname{erf}\left[\frac{\alpha i}{1-\alpha}\right]\right\}}{a d_{k, j}^{\alpha}+4 b \sin ^{2}\left(\frac{\sigma i}{2}\right)}\right| \leq 1 .
$$

Then,

$$
\frac{|f(j+1)|}{|f(0)|} \leq 1
$$

The property also holds for $j+1$. According to the inductive technique, the property is satisfied for any natural number. This completes the proof of Theorem 3. Theorem 3 shows that the Crank-Nicolson scheme is stable for the advection diffusion equation with the time fractional Caputo-Fabrizio derivative.

\section{Convergence analysis of the numerical solution}

Let us suppose that, at the point $\left(x_{i}, t_{j}\right)$, the exact solution of our considered equation is $P\left(x_{i}, t_{j}\right)(i=1,2,3, \ldots, N ; j=1,2,3,4, \ldots, M)$. We assume that the difference between the exact solution and the approximate solution at that particular point is provided by 
$\delta_{i}^{j}=P\left(x_{i}, t_{j}\right)-P_{i}^{j}$. The transpose matrix associated with the matrix $\delta_{i}^{j}=P\left(x_{i}, t_{j}\right)-P_{i}^{j}(i=$ $0,1,2,3, \ldots, N ; j=0,1,2,3,4, \ldots, M)$ is $\left(\delta_{1}^{j}, \delta_{2}^{j}, \ldots, \delta_{N}^{j}\right)^{T}$. However, the row $\delta^{0}$ is zero because it represents the initial condition. The recursive relation in connection with the CrankNicolson scheme for the time fractional advection diffusion equation is given as

$$
\begin{aligned}
& \left(a d_{k, 0}^{\alpha}+2 b\right) \delta_{i}^{1}+(c-b)\left(\delta_{i+1}^{1}+\delta_{i-1}^{1}\right)-\frac{Q_{i}^{1}-Q_{i}^{0}}{2 c_{\rho}}=R_{i}^{1} \quad \text { for } j=0 \\
& \left(a d_{i, j}^{\alpha}+2 b\right) \delta_{i}^{j+1}-\left(a d_{i, j}^{\alpha}+2 b\right) \delta_{i}^{j}+(c-b)\left(\delta_{i+1}^{j+1}+\delta_{i-1}^{j+1}\right)+(c-b)\left(\delta_{i+1}^{j}+\delta_{i-1}^{j}\right)-\frac{Q_{i}^{j+1}-Q_{i}^{j}}{2 c_{\rho}} \\
& \quad=-a \sum_{l=1}^{j-1} \delta_{i}^{j-l} d_{i, l}^{\alpha}+R_{i}^{j+1} \text { for } j>0 .
\end{aligned}
$$

The remainder term of the approximation for using the Crank-Nicolson scheme to solve the modified advection diffusion equation is given in this case as

$$
\begin{aligned}
R_{i}^{j+1}= & a P\left(x_{i}, t_{j+1}\right) d_{i, l}^{\alpha}+a \sum_{l=1}^{j} b\left(x_{i}, t_{j-l}\right) d_{i, l}^{\alpha}-b\left\{\left(P\left(x_{i+1}, t_{j+1}\right)-2 P\left(x_{i}, t_{j+1}\right)+P\left(x_{i-1}, x_{j+1}\right)\right)\right. \\
& \left.+\left(P\left(x_{i+1}, t_{j}\right)-2 P\left(x_{i}, t_{j}\right)+P\left(x_{i-1}, x_{j}\right)\right)\right\}+c\left\{\left(P\left(x_{i+1}, t_{j+1}\right)+P\left(x_{i-1}, x_{j+1}\right)\right)\right. \\
& \left.+\left(P\left(x_{i+1}, t_{j}\right)+P\left(x_{i-1}, x_{j}\right)\right)\right\}-\frac{Q_{i}^{j+1}-Q_{i}^{j}}{2 c_{\rho}}
\end{aligned}
$$

Now using the full approximation and considering the remaining terms, we obtain the following relation:

$$
R_{i}^{j+1} \leq D\left(2 k+h^{2}\right)
$$

Theorem 4 The Crank-Nicolson scheme for the advection diffusion equation with time fractional Caputo-Fabrizio derivative converges, and there exists a positive constant $D$ such that

$$
\left\|P\left(x_{i}, t_{i}\right)-P_{i}^{j}\right\| \leq D\left(2 k+h^{2}\right) \quad \text { for all }(i=0,1,2, \ldots, M, j=1,2,3, \ldots, N) .
$$

\section{Numerical simulation for different values of alpha}

In this section, using the new numerical scheme, we present the numerical simulation of the advection diffusion equation with the Caputo-Fabrizio derivative of fractional order for different values of alpha. We chose in this case $Q(x, t)=\sin \left[x+\frac{\pi}{4}\right], P(x, 0)=\cos [x]$, $P(0, t)=\cos [t], P(x, 10)=0, u=0.1, c_{\rho}=0.9, \varphi=3, \lambda=0.75$. The numerical simulations are depicted in Figures 1, 2, 3, and 4. It is worth noting that each figure represents the flow at scale alpha. It is very important to realize that fractional differentiation is able to control the variabilities of the plume movement within the geological formations. The pollution does not only move within a homogeneous medium but also within heterogeneous one; therefore, the plume paths cannot be predicted by the classical advection dispersion equation. In these figures, we can see that the proportionally of the density of pollution within the geological formation is not the same everywhere due to the heterogeneity, and 


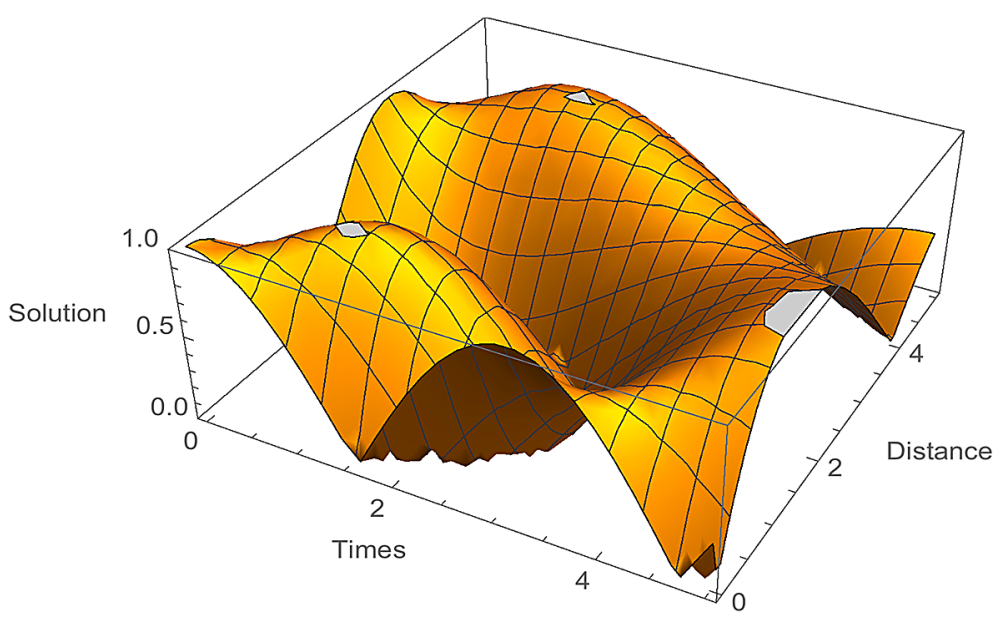

Figure 1 Numerical simulation at scale alpha $=0.15$

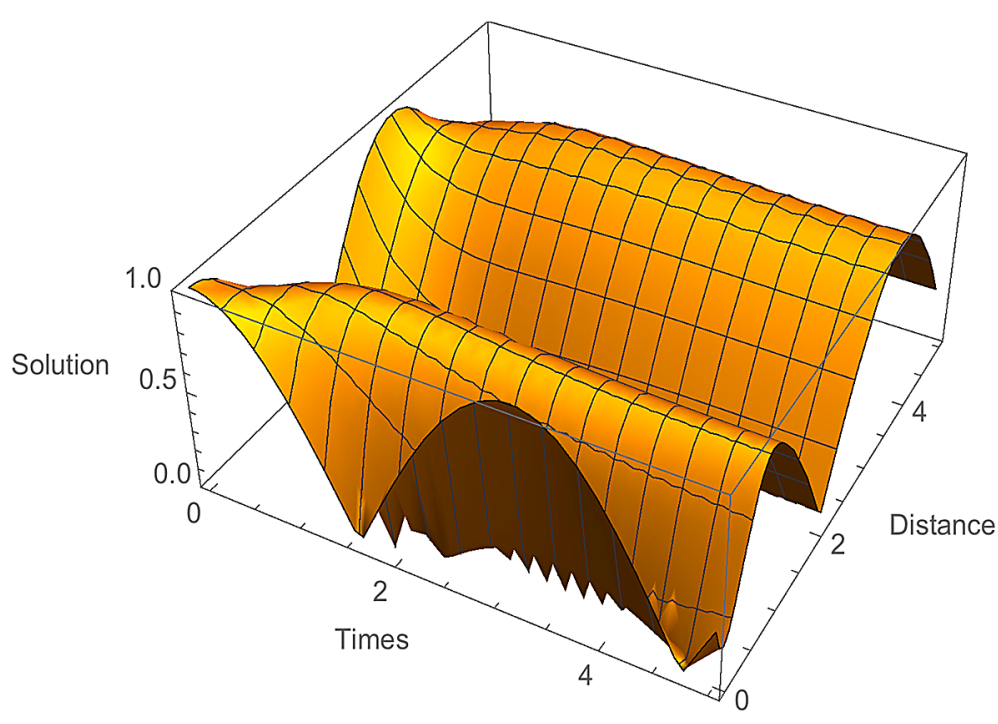

Figure 2 Numerical simulation at scale alpha $=0.55$

this is better described via the concept of fractional differentiation with nonsingular kernel.

\section{Conclusions}

We have proposed in this work the numerical approximation of the newly proposed derivative of fractional order in order to fit this derivative in the scope of numerical investigations. The new derivative is easy to use even numerically and display important characteristics that cannot be observed in the commonly used fractional derivatives. In order to test the possible application of the new numerical approximation of the new CaputoFabrizio derivative of fractional order, we presented a model of advection diffusion equation with the time fractional of the new derivative. We solved this equation numerically using the Crank-Nicolson technique. We showed the stability analysis together with some numerical simulations for different values of alpha. 


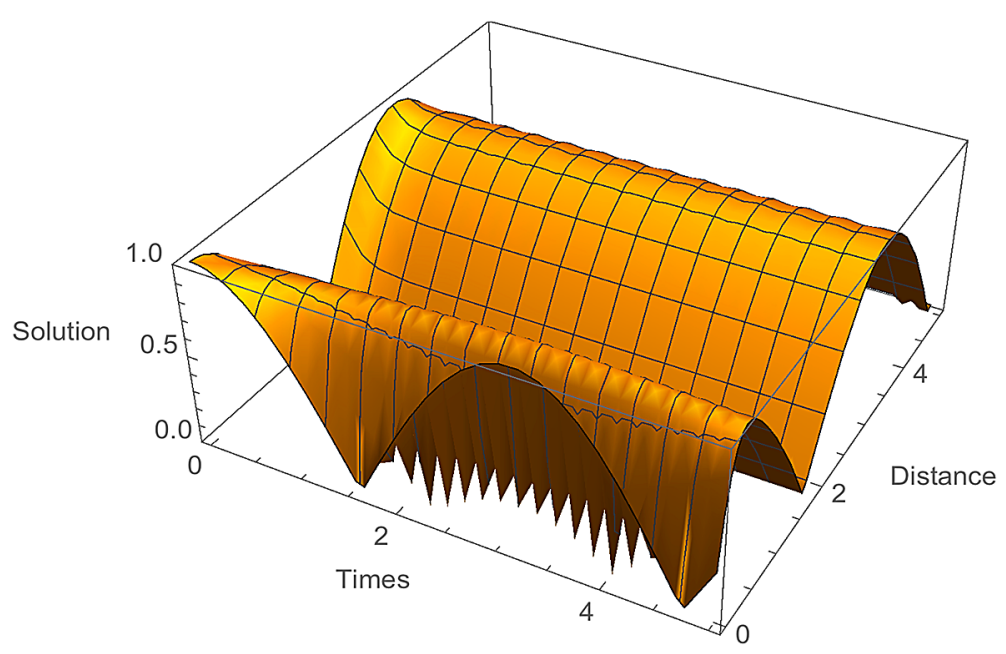

Figure 3 Numerical simulation at scale alpha $=0.85$.

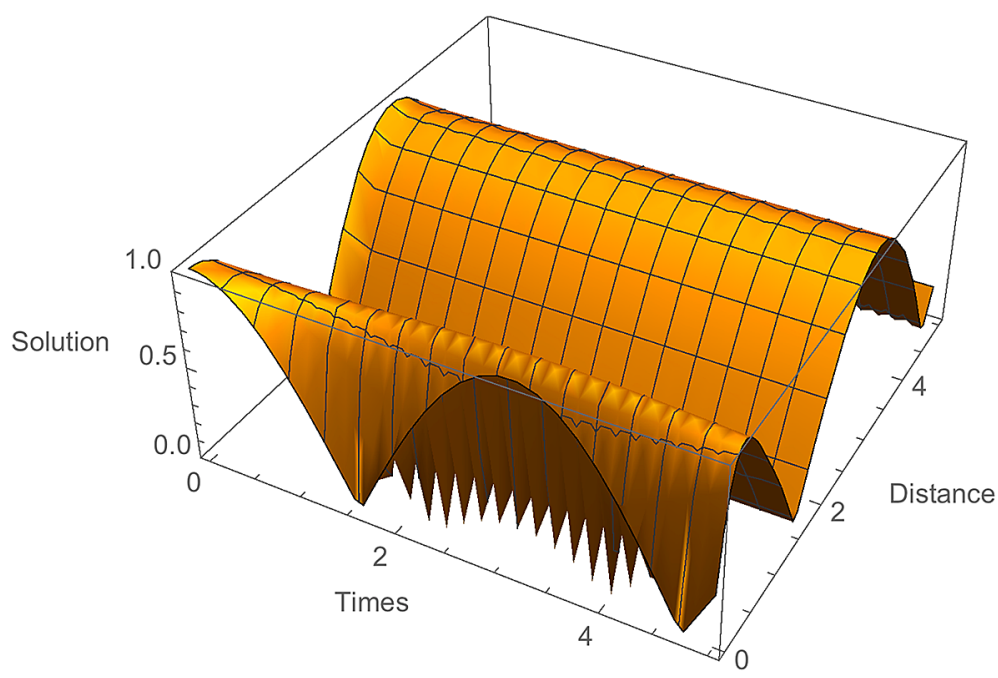

Figure 4 Numerical simulation at scale alpha $=0.95$

\section{Competing interests}

The authors declare that they have no competing interests.

\section{Authors' contributions}

The authors have equal contributions to each part of this paper. All the authors read and approved the final manuscript.

\section{Author details}

'Faculty of Natural and Agricultural Sciences, Institute for Groundwater Studies, Bloemfontein, 9300, South Africa.

2Department of Mathematics and Statistics, College of Science, Al-Imam Mohammad Ibn Saud Islamic University (IMSIU), Riyadh, 11566, Saudi Arabia.

\section{Acknowledgements}

We acknowledge the Editorial Board and the referees for their efforts and constructive criticism, which have improved the manuscript.

Received: 17 April 2016 Accepted: 24 May 2016 Published online: 14 June 2016

\section{References}

1. Caputo, M, Fabrizio, M: A new definition of fractional derivative without singular kernel. Prog. Fract. Differ. Appl. 1(2), 73-85 (2015) 
2. Losada, J, Nieto, JJ: Properties of a new fractional derivative without singular kernel. Prog. Fract. Differ. Appl. 1(2), 87-92 (2015)

3. Tian, Y, Wang, JR: On some Hermite-Hadamard type inequalities for convex functions via Hadamard fractional integrals. Prog. Fract. Differ. Appl. 1(2), 103-110 (2015)

4. Atangana, A, Nieto, JJ: Numerical solution for the model of RLC circuit via the fractional derivative without singular kernel. Adv. Mech. Eng. 7(10), 1-7 (2015)

5. Diethelm, K, Ford, NJ, Freed, AD: Detailed error analysis for a fractional Adams method. Numer. Algorithms 36(1), 31-52 (2004)

6. Li, CP, Tao, CX: On the fractional Adams method. Comput. Math. Appl. 58(8), 1573-1588 (2009)

7. Podlubny, I, Chechkin, A, Skovranek, T, Chen, Y, Vinagre Jara, BM: Matrix approach to discrete fractional calculus II: partial fractional differential equations. J. Comput. Phys. 228(8), 3137-3153 (2009)

8. Atangana, A, Secer, A: A note on fractional order derivatives and table of fractional derivatives of some special functions. Abstr. Appl. Anal. 2013, Article ID 279681 (2013)

9. Kilbas, AA, Srivastava, HM, Trujillo, JJ: Theory and Applications of Fractional Differential Equations. North-Holland Mathematics Studies, vol. 204. Elsevier, Amsterdam (2006)

10. Ashyralyev, A: A note on fractional derivatives and fractional powers of operators. J. Math. Anal. Appl. 357(1), 232-236 (2009)

11. Hanert, E: On the numerical solution of space-time fractional diffusion models. Comput. Fluids 46(1), 33-39 (2011)

12. Zhuang, $P, L i u, F, A n h, V$, Turner, I: Numerical methods for the variable-order fractional advection-diffusion equation with a nonlinear source term. SIAM J. Numer. Anal. 47(3), 1760-1781 (2009)

13. Lin, R, Liu, F, Anh, V, Turner, I: Stability and convergence of a new explicit finite-difference approximation for the variable-order nonlinear fractional diffusion equation. Appl. Math. Comput. 212(2), 435-445 (2009)

14. Crank, J, Nicolson, P: A practical method for numerical evaluation of solutions of partial differential equations of the heat conduction type. Proc. Camb. Philos. Soc. 43(1), 50-67 (1947)

15. Atangana, A, Oukouomi Noutchie, SC: Stability and convergence of a time-fractional variable order Hantush equation for a deformable aquifer. Abstr. Appl. Anal. 2013, Article ID 691060 (2013)

16. Akyüz-Daşciloğlu, A: Chebyshev polynomial solutions of systems of linear integral equations. Appl. Math. Comput. 151(1), 221-232 (2004)

17. El Tom, MEA: Application of spline functions to systems of Volterra integral equations of the first and second kinds. IMA J. Appl. Math. 17(3), 295-310 (1976)

18. Saeed, RK, Ahmed, C: Approximate solution for the system of non-linear Volterra integral equations of the second kind by using block-by-block method. Aust. J. Basic Appl. Sci. 2(1), 114-124 (2008)

19. Maleknejad, K, Karami, M: Numerical solution of non-linear Fredholm integral equations by using multiwavelets in the Petrov-Galerkin method. Appl. Math. Comput. 168(1), 102-110 (2005)

20. Meerschaert, MM, Tadjeran, C: Finite difference approximations for fractional advection-dispersion flow equations J. Comput. Appl. Math. 172(1), 65-77 (2004)

21. Doungmo Goufo, EF: Application of the Caputo-Fabrizio fractional derivative without singular kernel to Korteweg-de Vries-Bergers equation. Math. Model. Anal. 21(2), 188-198 (2016)

22. Doungmo Goufo, EF, Pene, MK, Mwambakana, JN: Duplication in a model of rock fracture with fractional derivative without singular kernel. Open Math. 13(1), 839-846 (2015)

23. Gómez-Aguilar, JF, López-López, MG, Alvarado-Martínez, VM, Reyes-Reyes, J, Adam-Medina, M: Modeling diffusive transport with a fractional derivative without singular kernel. Phys. A, Stat. Mech. Appl. 447, 467-481 (2016)

24. Gómez-Aguilar, JF, Córdova-Fraga, T, Escalante-Martínez, JE, Calderón-Ramón, C, Escobar-Jiménez, RF: Electrical circuits described by a fractional derivative with regular kernel. Rev. Mex. Fis. 62(2), 144-154 (2016)

\section{Submit your manuscript to a SpringerOpen ${ }^{\circ}$ journal and benefit from:}

- Convenient online submission

Rigorous peer review

- Immediate publication on acceptance

- Open access: articles freely available online

- High visibility within the field

- Retaining the copyright to your article 